\title{
Photocatalytic Degradation of Direct Orange Dye under Solar Light ${ }^{+}$
}

\author{
Madalina Elena David 1,2,*, Rodica-Mariana Ion 1,2, Lorena Iancu ${ }^{1,2}$, Ramona Marina Grigorescu ${ }^{1}$, \\ Ramona Elena Andrei ${ }^{1}$, George Ionut Radu ${ }^{1}$, Mircea Ioan Filipescu ${ }^{1}$, Alexandrina Nuta ${ }^{1}$ and \\ Ana-Alexandra Sorescu ${ }^{1}$ \\ 1 National Institute for Research \& Development in Chemistry and Petrochemistry - ICECHIM Bucharest, \\ 202 Spl. Independentei, 060021 Bucharest, Romania; rodica_ion2000@yahoo.co.uk (R.-M.I.); \\ lorenna77ro@yahoo.com (L.I.); rmgrigorescu@gmail.com (R.M.G.); andreiramona@hotmail.com (R.E.A.); \\ radugeorgeionut@yahoo.com (G.I.R.); mfilipescu3@gmail.com (M.I.F.); alexnuta1256@yahoo.com (A.N.); \\ anaalexandrasorescu@yahoo.com (A.-A.S.) \\ 2 Valahia University, Doctoral School of Materials Engineering, 13th Aleea Sinaia, 130004 Targoviste, \\ Romania \\ * Correspondence:madalina.david@icechim.ro \\ † Presented at the 15th International Symposium "Priorities of Chemistry for a Sustainable Development" \\ PRIOCHEM, Bucharest, Romania, 30th October-1st November 2019.
}

Published: 14 October 2019

Keywords: direct orange; dye; ferrite nanoparticles; photo-degradation

As the development of the textile industry has progressed, intense concerns have been focused on the contamination of the environment caused by dye pollutants, which can cause severe environmental pollution and health problems due to their variety, toxicity, and persistence characteristics. Therefore, in recent years, the degradation of dyes in industrial wastewaters has generated considerable attention due to the huge volume of production, slow biodegradation, low discoloration, and high toxicity [1]. This study was undertaken to determine the feasibility of oxidation processes in the treatment of textile dyes. Direct Orange 26 (DO-26) is an azo dye with potential ecotoxicity to exposed organisms [2]. Effective degradation of the DO-26 was studied by photocatalytic degradation under direct solar light.

The photocatalysts used in the study were $\mathrm{TiO}_{2}$, ferrite nanoparticles- $\mathrm{CoFe}_{2} \mathrm{O}_{4}$ and Fenton reagent. After the solutions were prepared, they were exposed to sunlight, between $2 \mathrm{~h}$ and $11 \mathrm{~h}$ in a Pyrex reactor of a cylindrical shape. Then, their maximum absorption at $495 \mathrm{~nm}$ and $519 \mathrm{~nm}\left(\lambda_{\max }\right)$ was recorded at specific times by a UV-Vis spectrophotometer.

Exposure of DO-26 under direct solar light in the presence of the catalysts caused important discoloration of the dye solution in a reasonably time. Conversely, the exposure of the DO-26 to solar light without catalysts did not cause any noticeable discoloration. It can be observed that in the first $2 \mathrm{~h}$, the absorbance of all DO-26 containing photocatalysts significantly decreased (Figure 1). For DO- $26+50 \% \mathrm{CoFe}_{2} \mathrm{O}_{4}+50 \% \mathrm{TiO}_{2}+\mathrm{Fenton}$ and $\mathrm{DO}-26+\mathrm{TiO}_{2}+$ Fenton, a tendency of decrease in absorbance after $11 \mathrm{~h}$ could still be observed. 

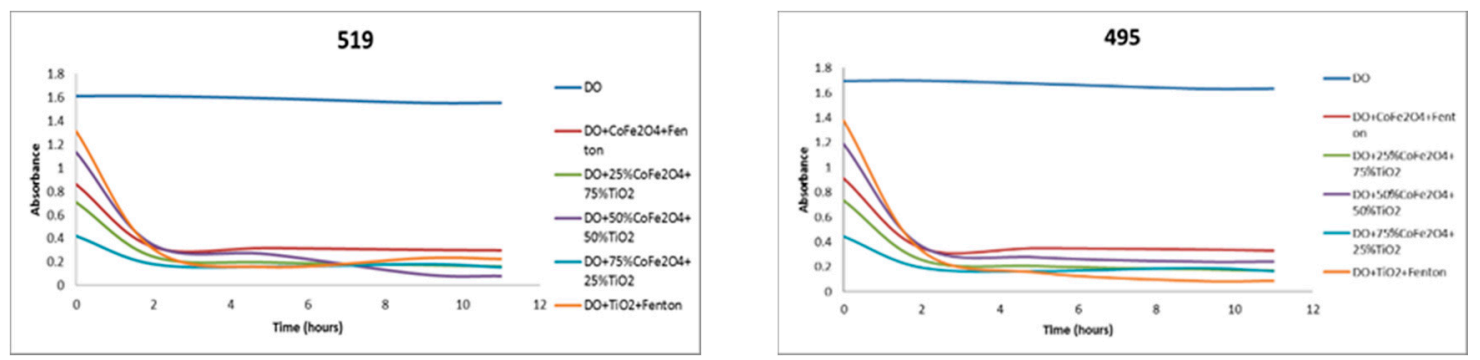

Figure 1. Photo-degradation of DO-26 under direct solar light.

This work demonstrates that these new materials are effective catalysts for the destruction of the industrial dye Direct Orange 26 (DO-26) under solar irradiation.

Acknowledgments: This work was supported by a NUCLEU Program, conducted with MCI support, project number PN.19.23.03.01.04.

\section{References}

1. Mehdipour Ghazi, M.; Azhdari, F. Photocatalytic degradation of textile dye direct orange 26 by using $\mathrm{CoFe}_{2} \mathrm{O}_{4} / \mathrm{Ag}_{2} \mathrm{O}$. Adv. Environ. Technol. 2016, 2, 77-84.

2. Amini, M.; Ashrafi, M. Photocatalytic degradation of some organic dyes under solar light irradiation using $\mathrm{TiO}_{2}$ and $\mathrm{ZnO}$ nanoparticles. Nanochem. Res. 2016, 1, 79-86.

(C) 2019 by the authors. Licensee MDPI, Basel, Switzerland. This article is an open access article distributed under the terms and conditions of the Creative Commons Attribution (CC BY) license (http://creativecommons.org/licenses/by/4.0/). 